\title{
PROBLEMS OF GASEOUS MOTION AROUND STARS
}

\author{
SU-SHU HUANG \\ Dept. of Astronomy, Northwestern University
}

\begin{abstract}
A distinction is drawn between radial and tangential modes of ejection from stars, and the possible flow patterns are described. They are: expanding streams, falling streams, jet streams, circulatory streams, and gaseous envelopes. Motion around Be stars is discussed at some length, as a preliminary to studying more complicated flow in binary systems. The rotational velocity of the Be star is insufficient to form the ring. It appears likely that radial instability is temperature sensitive. Rings and'disks in binary systems are discussed from the point of view of periodic orbits for particles within the gravitational field of such a system. The formation of these rings is discussed. The expected relation between rotational velocity of the ring and the orbital period is discussed. The relation of circumstellar streams to period changes is mentioned. Finally, the influence of magnetic fields on the circumstellar material and the system is discussed.
\end{abstract}

\section{Introduction}

Gaseous motion around stars is a very broad problem that can be studied from several points of view. In the past decade numerous papers have been presented in journals and at conferences that have been devoted solely to this subject. It is not my purpose to give a general review of the entire field. Indeed so many papers have appeared that a review by a single person cannot do justice to them all. Rather I will limit myself only to those topics within the general scope of gaseous motion around stars that I myself have been interested in since the days of my close association with the late Dr. Struve who had greatly influenced me in putting the emphases of theoretical studies on the observational relevance, a practice I have followed in this presentation.

In what follows we will first classify in Section 2 both ejection modes and flow patterns according to a kinematic consideration because what can be directly observed is nothing more than kinematics of gaseous motions. Three problems will then be discussed: (1) the emission problem of Be stars, (2) rings and disks in binary systems, and (3) magnetic braking and its consequences respectively in Sections 3, 4, and 5.

\section{Kinematic Modes of Ejection and Flow Patterns}

\subsection{EJECTION MODES}

One of the important problems associated with mass loss from stars is the ejection mechanisms. We shall divide them into three kinematic modes: (1) the pure mode of radial ejection, (2) the pure mode of tangential ejection and (3) the mixed mode.

\subsubsection{Radial Mode of Ejection}

The radial mode of ejection extends over a wide range from the solar wind to nova and supernova outbursts. It may be caused by several factors, like (1) a high temperature and pressure layer such as the solar corona in the case of a solar wind (Parker, 1963) or for that matter the stellar wind, (2) a hot and expanding photosphere as in the case of the 
nova explosion (McLaughlin, 1943, 1950; Pottasch, 1959; et seq.), (3) radiative instability as has been suggested by Lucy and Solomon (1970), (4) magnetic and other kinds of instability that may occur in the outer layers of the star, (5) overshots of current elements in the convective zone in stellar atmospheres, or (6) the simple process of evaporation from the surface.

We have considered the radial ejection only in a statistical sense. For example take the simple case of evaporation, which has been invoked by Jeans (1925; also Spitzer, 1952; Öpik, 1963) in explaining the escape of mass from planetary atmospheres. In the very top layers of the atmosphere where collisions are infrequent, particles with velocities greater than the escape velocitiy will get away from the atmosphere for good. Since the thermal velocities of particles are randomly oriented in space, few trajectories of the escaped particles actually follow the radial path. However, when all escaped particles are considered together, the motion is statistically radial if the planet neither rotates nor possesses a magnetic field.

Of the six cases of the radial mode of ejection all except the last three cases can be treated by Parker's theory of the solar wind or its variations.

\subsubsection{Tangential Mode of Ejection}

The pure mode of mass ejection through the tangential motion can be envisaged in connection with the gravitational contraction of a rapidly rotating body. It was pointed out by Laplace in his theory of formation of the solar system that a nebula with a net angular momentum will flatten itself first in a lenticular form in the process of gravitational contraction. Eventually the outer ring of this lenticular body will detach itself from the central part because of the latter's further contraction. In this way the matter left the central body without the aid of radial motion. Hereafter we shall call it the Laplace mode of mass ejection. Obviously the Laplace mode of detachment is related only to the gravitational contraction of a rapidly rotating body. It does not involve any change of kinematic state of the ejected mass at the equator. Hence no ejection theory is required.

Another kind of the pure tangential mode of ejection is through the action of viscosity which transports the angular momentum from the inner layer of the atmosphere to the outer region, thereby creating rotational instability at the equator. The viscosity may be molecular, photon, turbulent or magnetic. A general review of the effectiveness of these different causes of viscosity has been given by Limber and Marlborough (1968).

\subsubsection{Mixed Mode of Ejection}

The two pure modes described previously represent only an idealization. Actually most cases of ejection have both radial and tangential velocity-components at the time of ejection. In the first place ejection of mass by stars in the binary system follows necessarily the mixed mode simply because of lack of spherical symmetry: Also, in the presence of rotation and/or magnetic field, the solar and stellar winds are no longer purely radial. They represent interesting cases of mass ejection in astrophysics (e.g. 
Limber, 1967). We shall make no attempt to present a general survey of this mixed mode of ejection but will select a few relatively simple cases in this category for discussion here, because it is only through a complete understanding of simple cases that the more complicated cases can be meaningfully tackled.

\subsection{Flow PATternS}

Gaseous motion in an extended medium can take place in an infinite variety of ways. Look at the wind on the surface of the Earth. At any time there are global wind systems, local wind systems and what may be called microwind systems that you find in the neighborhood of tall buildings. It is hopeless to examine them in all their details. This has made meteorology one of the most frustrating branches of science and the weather forecasting one of the unreliable scientific predictions. But the meteorologist who can set up stations at different locations for direct measurements is lucky when compared with the astrophysicist who can only observe the entire system of gaseous motion around the star from a single remote station many parsecs away. That we have learned something about geaseous motions around the star must be attributed to the tenacity of astrophysicists, like Dr. Otto Struve, who has persistently searched for the mystery of the stellar world in spite of this handicap.

I cite this meteorological example in order to emphasize the simple truth that our knowledge of the gaseous motion around the star cannot be complete. We will be lucky if we can discern some general patterns of motion. With this point of view in mind we can discuss three basic modes of motion of gases after they have been ejected from the star. They are:

(1) General expanding streams characterized by the P Cygni profile, WR emissions, and the solar wind.

(2) General falling streams to the star from which the matter originally came, or to the companion in the case of a binary system such as that suggested by Huang (1963a) for the observed emission in $\beta$ Lyrae.

(3) Jet streams that are confined to a limited region of space and have perhaps much higher densities than the general medium, such as those suggested by Struve (1941) for interpreting the satellite lines observed in $\beta$ Lyrae and by Batten (1969, 1970; also Batten and Plavec, 1971) for interpreting the observed emission in U Cephei.

(4) Circulatory streams characterized by the gaseous ring in Be stars - a problem that will be extensively discussed later in this paper.

(5) Envelopes characterized by random motions without systematic motions. Because there is no containing wall in channeling the motion of ejected matter, the latter will inevitably diffuse into the entire vicinity around the star, creating what we may call an envelope. In addition to diffusion the collisions of streams also help the creation of the envelope. This situation may be seen by considering a room full of smoking people, with each puff producing a little stream. Soon the entire room is filled with smoke through diffusion of particles as well as mixing of different streams. However one can still discern the little streams of smoke coming out of each smoker's mouth even when the room has already been filled with smoke, provided that the density of smoke particles in the 
general background is less than that in the stream. In such a case we must distinguish two substrata - those in the general background and those in the streams when we study the motion of smoke particles in the room. Similarly when we discuss the gaseous motion around the star, we must also consider in most cases these two substrata: the envelope characterized by the random motion and the streams characterized by the systematic motion.

Under this consideration the evelope may be identified with what Batten (1970) calls 'clouds'. However if we imagine in our example of smoking that the density of smoke particles streaming out from the smoker's mouth is much less than that already present in the room, the stream would hardly be distinguishable. While such an imagined case does not actually happen in the smoke-filled room because someone will open the window long before this state of affairs would occur, it is quite possible that the density of the stellar envelope becomes so high that the streams can no longer be traceable. They mix with the envelope immediately after being ejected from the star. This situation can happen when the star is massive and its strong gravity holds the matter there. In this way the star develops a dense envelope. Perhaps the extended atmospheres such as in Zeta Aurigae stars are envelopes in this sense. The solar chromosphere and the inner part of the solar corona may also belong to this classification, because some kind of mass ejection, in addition to the well-known mechanism of simple energy transfer, must be taking place below the chromosphere in order to replenish the mass that is being continuously lost from the outer corona in the form of the solar wind.

In summary the motion of ejected matter in the stellar neighborhood can be basically divided into two substrata (1) one with completely random motion and (2) one with somewhat systematic motion or motions. The great variety of cases of gaseous motion that will be actually observed in stars arises from (1) densities and flow velocities in the streams with respect to the density and random velocities in the envelope and (2) the different nature of streams. Also these two substrata - occupying the same region in the stellar neighborhood are continuously interacting with each other. Such an involved state is further complicated by the fact that the ejection of mass by the star may not be a continuous and steady process. All these factors contribute to the time and spatial variations of observed motions.

Around single stars the envelope may have an axial symmetry even perhaps only in a statistical sense. But in the binary system the envelope must be quite complicated. Since space around a binary system can be naturally divided into three regions by the innermost contact surface (Kuiper, 1941) - one around each of the two component stars and one around the entire system, three envelopes can in principle exist with each being confined to one region of space. However that does not mean that the envelopes are separated by empty space. Rather they denote only regions of relatively high densities separated by regions of relatively low densities. Because of the geometrical and physical nature of the stars some binaries may have less than three such envelopes or none at all. In any case the non-spherical distribution of matter in the envelopes in the binary system may produce phenomena that change with phase, although such changes 
could be caused by many other factors, such as gravity, reflection, etc. recently considered by Buerger (1969) that are not related to the envelopes at all.

In what follows we often discuss the problems of the gaseous stream for the sake of simplicity without considering the presence of the envelope although the motion is actually observed in the general background of the envelopes as has been already emphasized. We shall consider mainly some problems connected with the circulatory mode of motion - topics that are closely related to the transport of angular momentum from the star to the gaseous stream. In addition we will also discuss the reversed problem of the effect of angular-momentum transport in the ejection process, on the star itself especially in the presence of stellar magnetic field.

\section{Gaseous Motion Around Be Stars}

It is our point of view that in order to understand the complicated cases of the gaseous motion in the binary system, it is helpful to learn first the relatively simple case of the gaseous motion around the single star. For this reason we shall consider here one of the simplest cases of the gaseous motion, namely the circulatory motion around single Be stars.

\subsection{MODE OF EJECTION}

While it appears to be trivial to divide the ejection modes into the three kinds mentioned previously, it nevertheless gives us a new insight to some interesting problems. Struve (1931; also Crampin and Hoyle, 1960) has proposed that the gaseous ring is associated with many a Be star and is the source of its emission lines and further assumed that the ring was formed according to the Laplace mode of mass ejection. Actually it is easy to see that this cannot be so. Let us first consider the time scale. The gravitational contraction of a star is a slow process. The time scale is measured in terms of thousands of years. The behavior of the emission ring is not consistent with such a slow process. It appears and disappears in terms of decades and its intensity varies within hours or even minutes. Such rapid changes suggest that the ring cannot be formed by the slow and gentle detachment of matter according to the pure Laplace mode.

Secondly, Slettebak (1966) has found that the largest observed rotational velocities for stars of spectral types from 09.5 to F0 of luminosity classes IV-V are always below the computed equatorial breakup velocites. How, then, could the gaseous ring be formed purely by the Laplace mode of ejection. Slettebak mentioned radiation pressure and macroturbulence as the possible cause for this discrepancy. Of course, the presence of a stellar magnetic field can also change the picture (Hazlehurst, 1967; Limber and Marlborough, 1968). However, because of the high efficiency of angular momentum transport through magnetic fields, it is likely to help dissipate the gaseous ring instead of maintaining it. Another way of looking at the discrepancy has been advanced by Hardrop and Strittmatter (1968) who gave reasons to suggest that the observed rotational velocities might have been underestimated. According to them the most rapidly rotating stars are indeed at the point of rotational breakup at the 
equator. However, this explanation cannot quantitatively remove the discrepancy. Therefore we are inclined to agree with Limber (1970) that a star need not be rotationally unstable at its photosphere in order to show the Be phenomenon. In other words, the ring is not formed by the pure tangential mode.

Finally we may point out that many single A-type stars rotate as rapidly as B-type stars, but few, if any, show the emission feature that can be attributed to the gaseous ring. Of course one may argue that this could be due to a difference in excitation by B- and A-type stars. However, such as argument meets a serious difficulty in the fact that the emission rings have been found around A-type, and occasionally even F-type stars in binary systems. Among 15 such systems collected by Sahade (1960), twelve stars around which the gaseous ring revolves are of spectral type A and one of spectral type F. Only two are B stars. This shows not only that A stars but also F stars can easily excite the emitting atoms in the gaseous ring. It follows that the deficiency of gaseous rings around the single and rapidly rotating $\mathrm{A}$ stars is intrinsic and deserves a close examination (Huang, 1972).

From what has been said, it is clear that formation of the gaseous ring around $\mathrm{Be}$ stars is not due purely to rapid rotation of the star. There must be involved some radial instability in addition to rapid rotation. Whatever is the cause of the instability, it creates a radial component in the ejection velocity that facilitates the formation of rings.

From the observed fact that few rings have been found around single A-stars in rapid rotation, and may around single $B$ stars, we may suggest that the radial instability is temperature sensitive. If we measure such an instability, whatever is its nature, by assigning a radial component for the ejection velocity, it may be said that this component increases with the temperature of the star. Hence in the A-stars this radial component must be very small or non-existent so that it playes little part in influencing the rotating matter at the stellar equator. Hence the ring is not formed. But in the B stars the velocity component contr.buted by radial instability must be large enough in lifting up the matter in the equator to become a detached ring that produces emission lines. As the temperature within the range of B-type stars increases, the frequency of occurrence of emission lines increases correspondingly. While only a fraction of one percent of B9 and early A-type stars show emission lines, this figure increases to $1 \%$ for B8 stars, 5\% for B5 stars and 10-15\% for early B-type stars (Curtiss, 1926; Merrill and Burwell, 1933). Although these data are more than $30 \mathrm{yrs}$ old, and a new and refined statistical study of the percentage of Be stars in different sub-divisions is highly recommended, we believe that this general trend will not be changed by any new statistical study. Thus such a general trend confirms the temperature-sensitiveness of the process of ring formation.

When the temperature of the star further increases, the radial component of the ejection velocity will become even greater. Eventually this would make the resultant velocity so high that the ejected matter can escape from the gravitational attraction of the star. If we assume that the star rotates so rapidly that the matter at its equator is on the verge of rotational breakup, the condition of matter escaping into infinity will 
be met when the radial component of ejection velocity is equal in magnitude to the rotational velocity at the equator. For stars which rotate with less rapidity, the radial component will be somewhat higher than this value before the condition of escape is reached. Thus the range of the radial component of ejection that may lead to the formation of gaseous rings is not very wide. This may explain why the gaseous rings around single stars exist dominantly around those of spectral type B and the frequency of occurrence of rings declines when the star becomes hotter. That does not mean, of course, that emission will disappear in very hot stars. On the contrary a higher percentage of $\mathrm{O}$ stars shows emission than that of B stars. Only the nature of the emission is changed. For $\mathrm{O}$ stars emission will dominantly come from the amorphous gas that moves away from the star instead of arising from the rotating ring. Indeed S Pup, an O5f star, shows the absorption line at $\lambda 1175$ of $C$ ill shifted to shorter wavelengths by about $5 \AA$ with an emission edge in the normal position (Carruthers, 1968).

In summary the formation of gaseous rings around Be stars results from two events that simultaneously go on in the star, rapid rotation and the radial instability. In $\mathrm{A}$ stars either radial instability is weak or non-existent, one condition for ring formation is missing. Therefore few gaseous rings have been observed around single A stars. In $\mathrm{O}$ stars the radial instability is so strong that the resultant velocity of ejection becomes greater than the escape velocity. Therefore we see the decline of gaseous rings but at the same time a rise of the frequency of emission in hotter and hotter stars. From this view the emission resulting from the ring and that resulting from the expanding gas form a group differentiated only by the relative importance of the tangential component versus the radial component of the ejection velocity. The temperature dependence of the radial instability as inferred from this discussion seems to agree with what has been suggested by Lucy and Solomon (1970) as due to the radiation pressure.

Lucy and Solomon's theory of mass flow is based on a consideration of radiation pressure. The absorption in the ultra-violet resonance lines of ions such as Si IV, $\mathrm{C}$ IV, $\mathrm{N}$ v, and $\mathrm{S}$ vi that are located in the intensity peak of stellar continuous radiation creates negative effective gravities in the outer parts of the reversing layers of hot and luminous stars. As a result a static reversing layer is untenable and a radial flow results. Their theory applies to Of and early supergiant stars and explains satisfactorily the P Cygni profiles observed in the far-ultraviolet spectra of these stars. According to their calculation B stars close to the main-sequence are located just near the edge of the instability region in the $\log g$ - $\log T$ diagram, where $g$ and $T$ denote respectively the surface gravity and the temperature in the atmosphere. If the star rotates rapidly, it can easily enter into the region of instability, because rotation further reduces the effective gravity. Thus, it appears that the phenomenon of gaseous ring around Be stars is due to a right combination of rotation and absorption in resonance lines

Lucy and Solomon have followed Parker's theory of the solar wind in their development of mass flow from the star. They can therefore explain the high velocities reaching several thousand $\mathrm{km} \mathrm{s}^{-1}$ as observed. However, in the case of Be stars the problem is slightly different. In the first place there is the rapid rotation of the star. While the stellar wind of a rapidly rotating star has been studied by Limber (1967) it 
may not be applicable to the present case, because what we observe here are rotating gaseous rings but not stellar winds. Whether superimposed on the ring structure there is also a stellar wind from the star is at present not clear.

\subsection{MODE OF GASEOUS FLOW}

A simple consideration indicates that the specific angular momentum of a particle rotating in a circular orbit under the gravitational force of a central mass is proportional to the square root of the orbital radius. Consequently even at the point where the star is rotationally unstable at its equator, there is not enough angular momentum to lift up the mass at the equator in order to form a detached rotating ring. Consequently in the absence of magnetic field or other agencies that transport angular momentum, only a fraction of matter that is lifted up from the equator can go into the rotating ring. If the radius of the star at the equator is $r_{0}$, and the radius of the ring is $r_{1}$, the fraction of the matter that goes into the ring is $\left(r_{0} / r_{1}\right)^{1 / 2}$, if the rest either falls back into, or escapes from, the star, carrying away no net angular momentum. Actually the fallen and escaped gas may carry away some amount of angular momentum, hence the actual fraction of ejected matter that goes into the rotating ring is likely less than $\left(r_{0} / r_{1}\right)^{1 / 2}$.

Thus we see that during the formation of the gaseous ring we expect to observe some mass either falling into or escaping from the stars. Perhaps the two simultaneous events - the gaseous ring and the falling matter - might have led Hutchings (1970, also Crampton and Hutchings, 1972) to suggest the idea of falling rings because observationally the two separate events of gaseous rings and falling matter on the one hand and the single event of falling rings on the other are not greatly different. In general we may also expect to see some matter escaping from the star. Whether the escaping matter takes the form of the stellar wind, or follows the process of evaporation cannot at present be answered either by observations or by theory. Also the question of what fraction of ejected matter is going to become a gaseous ring, what fraction is falling and what fraction is escaping is not known. Suffices it to say one of the controlling factors remains the radial and tangential components of the ejection at the stellar surface.

Thus we may conclude that the mixed mode of mass ejection from a rotating star in the absence of stellar magnetic field leads in general to several substrata of mass around the star in the equatorial region. There are (1) the ring or disk structure and (2) the falling and/or expanding substratum and finally (3) the general envelope supported by random motion. Away from the equatorial region, matter is ejected if at all at a much less rate than at the equator on account of smaller tangential velocities. Several possibilities exist for the ejected matter. It may escape into infinity, fall into the star or into the disk in the equatorial plane or stay in the stellar neighborhood to form an envelope. What course it actually follows obviously depends again upon the rate of rotation and degree of radial instability. Perhaps studies of pole-on stars such as one made by Togure (1969) may provide us with some empirical information. 


\subsection{INTERPRETATION OF EMISSION VARIATIONS}

It may be argued that any new ejection of matter by the star disturbs the ring, because the newly ejected particles can knock the particles in the ring out of the orbits. This may explain the frequent fluctuation of the intensity, and disappearance and re-appearance of emission lines produced by the gaseous ring. However, the fact that emission rings are observed at all means that ejection of matter by the star is likely intermittent or if there is any continuous ejection, it must be exceedingly mild because each new violent ejection will likely destroy the existing ring. Once the ring has been destroyed the matter may or may not settle down into a new ring, which may or may not be identical to the previous one thereby creating a variety of possibilities that are to fascinate the observer. The observed result seems to show that the intervals between bursts are astronomically short and perhaps irregular. Whether the quiescent interval before, and the strength of, each burst follow some rule is a question that has not been extensively studied. Intuitively we may conjecture that the strength of each burst is likely to increase with the quiescent interval that precedes it.

The variation in the emission may be either irregular or roughly cyclic for a certain period of time and may take place either in the line profile only or in both the profile and the equivalent width. The cyclic variations themselves are complicated because periods can vary from decades to minutes or perhaps even seconds. Obviously we do not expect to find all variations due to the same cause. What we actually observe is of course the combined result arising from all causes. Thus our purpose is to disentangle and isolate various causes and treat them individually. In general, the variation in the profiles without the change of the total strength is most likely due to the effect of geometry, just like the effect of rotation on stellar absorption lines (e.g. Huang and Struve, 1960). Such a variation is comparatively easy to understand because it can be studied without resorting to the physical cause of the emission. It is this group of variations that will give us some information about gaseous motions around the star. The change in the profiles of this nature likely means the reshuffling of radial velocities of emitting atoms without any change in their total number.

We will now discuss several kinematic problems that can produce some kind of observed variations in emission. First, there is the well-known $V / R$ variations with periods stretching from years to decades. While the relative intensity of two components of emission varies, their total strength seems to be roughly constant. Theories for this kind of variation were last reviewed by McLaughlin (1961), who has devoted his time and energy in studying this kind of variations more than anyone else. $\mathrm{He}$ reviewed three models, namely (1) the rotating-pulsating model (2) the intermittently expanding-rotating two-layer model and (3) the eccentric rotating model.

The artificiality of the first model is obvious. McLaughlin has pointed out several defects from the observational point of view. Theoretically it is difficult to see pulsations of such long periods taking place in the neighborhood of stars on or close to the main sequence. Also, there is no conceivable mechanism to drive the pulsation of a 
tenuous detached ring which, being maintained simply by the angular momentum, possesses no elasticity of its own.

MćLaughlin's second model assumes two layers - the inner emitting and the outer absorbing layer. By considering the cyclic change of the motion in both layers it is possible to obtain the $V / R$ variation. McLaughlin has found that the observed facts are incompatible to this model. Theoretically it is hard to understand how two layers can maintain their separate existence cycle by cycle.

In the eccentric rotating model the $V / R$ variation is attributed to the apsidal motion of a rotating elliptical ring of emitting gases. This possibility was first mentioned by Struve (1931) but it was McLaughlin who seriously embraced it and made some preliminary explorations. Indeed both $V / R$ variations and velocity shifts of emission and absorption components based on this model seem to be consistent with observed results. Also Johnson (1958) examined the adequacy of the star's oblateness as the cause of the apsidal motion of the elliptical ring and found the rate of this motion indeed adequate for explaining the observed periods of $V / R$ variations. We have made a quantitative study of the change in emission profiles based on this model. Since the observed $V / R$ variations are related to the eccentricity of the elliptical ring, the latter can therefore be determined empirically. It appears that in most cases a small eccentricity is enough to account for the observed amplitude of $V / R$ variations (Huang, unpublished). Also according to this model the absorption line remains always in the center of two emission edges as observed.

Rapid variations of emission profiles in Be stars have been found recently by many investigators like Hutchings and associates here in Victoria, Bahng in Northwestern University and a large group in Meudon. The emission may vary either in the profile only or in both the profile and the strength. Obvoiusly no single cause can be assigned to such a heterogenous group of variations.

One of the possibilities may be the non-uniform distribution of emitting gas in the ring (Huang, 1972). It can be easily seen that even in the circular ring, such a distribution produces in the emission line not only asymmetry but also time-dependence. The variation is due to the fact that the emitting particles revolve with different Keplerian velocities on circles of different radii. If the gas is tenuous, it produces rapid variation in profiles but no change in the total intensity of the line. Such a variation may or may not show any periodicity. Even if it should indicate some periodicity it does not repeat itself exactly in every cycle. On the other hand it has a definite tendency of being damped due to the smoothing effect on the uneven density distribution in the ring by both differential and random motions. The time scale of damping depends upon the width and the size of the ring as well as the mass of the central star.

Another possibility of having asymmetric profiles and time variations may result from the multi-substratum nature of the medium around the $\mathrm{Be}$ star mentioned previously. The size of the rotating ring which is fixed by its angular momentum cannot change easily without angular momentum transfer. However, there are other substrata of falling, escaping and/or amorphous gas. The emission arising from these substrata will modify the emission due to the ring. As a result the resultant emission 
will be asymmetric. As has been mentioned before the phenomena that have been interpreted by Hutchings (1970) and by Crampton and Hutchings (1972) as due to falling or expanding rings result, according to the present model, from the multisubstratum nature of the gaseous medium.

\subsection{TIME SEQUENCE OF RING FORMATION}

Why is the ring elliptical in some cases and circular in others? Why is the density in the ring not uniformly distributed? Why does there exist the other substrata in addition to the ring? All these questions lead to the fundamental problem of mass ejection from the star. Observations show that the ejection must be intermittent. Each new violent ejection destroys the previous ring structure if it exists and makes the medium chaotic and full of large turbulent eddies. In this initial phase there is no structure in the medium even in the equatorial plane. The emission is likely strong and complex. Not only the profile but also the total strength may vary greatly because of the violent nature, such as collision of eddies, shocks, etc., in the medium. However, because of the angular momentum, some of the matter may gradually settle down to form a ring while the rest may fall into the star and/or escape into space. This is the multisubstratum phase we have emphasized before. In this second phase the emission can also have various kinds of variations. Falling and escaping components in the substratum will be dissipated first. Eventually even the amorphous component will be dissipated, leaving in the final phase only the ring itself until it is destroyed by the next violent ejection from the star or by gradual dissipation. During the final phase variations in the emission are expected only in the profile.

According to this scenario it is easy to see that the density in the ring cannot be uniform when it is formed out of the chaotic medium. Also some small ellipticity could be expected in the ring. If, however, the ring should be left alone for a long time without any disturbance, it will eventually reach a circular state, as are Saturn's rings. But around the Be star, the ring is never left alone for any long period of time. That is why we observe so many variations in the emission lines. Conversely these variations indicated how active Be stars are.

The characteristic feature of our model that differs from other ones is its developing nature. It does not advance any single supporting mechanism for the envelope. Indeed from the comprehensive study by Limber and Marlborough (1968), it is quite evident that no single mechanism can satisfactorily explain how the envelope can be maintained. Although they have concluded that the support is centrifugal, as most previous investigators since Struve had done, they have found difficulty in finding how such a support can be achieved through thermal and radiative viscosity and have had to resort to turbulent and/or magnetic viscosity. At the same time they have further pointed out that if the turbulent viscosity is responsible for transporting the angular momentum it has to be supersonic. Whether such turbulence can develop and is consistent with the observations is not clear. Therefore they have concluded that the "magnetic field remains a prime candidate for the required viscous agent even though the case for its importance is largely circumstantial". 
The idea of magnetic transport of angular momentum has also been taken by Hazlehurst (1967) in his theory of ring formation. He follows the concept of magnetic braking mechanism advanced by Lüst and Schlüter (1955; also Mestel, 1959). While the idea is attractive we must point out that all magnetic theories for ring formation would predict that the ring should be formed around not only rapidly rotating stars, but also moderately rotating stars. This is because magnetic field is a very efficient agency for transporting angular momentum from the star to the envelope. But the observation shows evidence that only rapidly rotating stars possess rings. For this reason we are uncertain whether the magnetic field plays a role in the ring formation around Be stars.

In our picture we consider the medium that evolves with time. During the first two stages the envelope is not in the hydrostatic equilibrium. Consequently the questions of supporting mechanisms does not arise. In this first stage the medium is dominated by the ejected matter from the star. In the second stage, the envelope adjusts itself after the disturbance suffered in the first stage. Some particles are supposed indeed to fall into the star, while others remain in the envelope. Only in the final stage, we assume the stage of the centrifugal support. Because of what happens in the second stage, we have achieved the centrifugal support in the final stage, without resorting to the magnetic or other kind of viscosity.

What makes the ejection of matter from the star intermittent instead of continuous which has been seen in the observation and accepted in our model, is perhaps due to the nature of marginal instability. Once instability is relieved by ejection of matter, it may take some time to rebuild the potential for the next instability. For example, after a large amount of angular momentum is lost in an outburst, the equatorial matter may become stable for a while until its rotation is accelerated by a little more of the contraction of the core of the star (Crampin and itoyle, 1960) and the transport of angular momentum outward (Limber, 1969). Perhaps a small change in the rotational velocity at the equator will tip the balance of stability and instability, because the change affects not only the tangential velocity but also the effective gravity that controls the vertical instability. Long-period variations of emission sometimes can last a few cycles indicating that the ring can persist without much disturbance several tens of years. Other events such as disappearance and reappearance of rings seem to confirm that the interval between two consecutive violent eruptions is also of this same order of magnitude. Tentatively we adopt this value as the average time scale for building up the instability. Obviously the actual time scale varies from star to star.

In this discussion we have emphasized only the qualitative nature of the evolution of the gaseous medium around Be stars. It is very difficult to treat the first two phases because of the time-dependent nature of the entire envelope. The envelope is at first chaotic, its motion being dominated by the ejection mode which can not at present be quantitatively described. In the process of ring formation, it dissipated matter as well as energy belonging to the random motion. It is unlikely that one can express this situation in any unique way. Perhaps a large number of plausible models may be built 
for different stages of development in this second phase. One of such models has been given by Limber(1969).

\section{Rings and Disks in Binary Systems}

\subsection{Mathematical CONSiderations}

What has been said so far from single stars may not all be applied to stars in binary systems. Because of the orbital motion, it becomes meaningless to consider the pure radial or pure tangential mode of ejection. For the same reason the gaseous flow in the binary system is more complicated than that around the single star. By considering the topographical structure of the equipotential surface in the binary system, Kuiper (1941) pointed out the importance of gaseous flow through the Lagrangian points, a concept that proves to be extremely useful in our interpretation of the observed phenomena in binary stars. Following Kuiper, who treated the gaseous flow within the framework of the restricted three-body problem, many investigators have computed numerous orbits that a particle can move in a binary system. However, the value of such calculation has been questioned because the three-body calculation necessarily neglects collisions among the gaseous particles. If one should take a look at some single trajectory with its many loops and cusps, it is quite clear that the neglect of collisions is most unrealistic. Also there are an infinite number of trajectories one can compute. How could they be interpreted in terms of actual flow? For these reasons Prendergast (1960) has tried to study this problem from the hydrodynamical consideration. He has written down the appropriate hydrodynamic equations, but made no attempt to discuss how suitable boundary conditions may be imposed to the differential equation of flow.

Thus we see that the approach based purely on the restricted three-body problem suffers the difficulty of neglectıng collisions and the hydrodynamic approach encounters the unsurmountable problem of how to impose suitable boundary conditions that are consistent with the physical reality. By neglecting the pressure and by making some approximation Prendergast succeeded in obtaining a particular solution depicting circulatory flows around each component and around the entire system. However, it does not tell you how such a flow can be achieved. Consequently it does not provide the answer to the question of when and why a gaseous ring is expected around the more massive component, the less massive component, or each of both components, in a particular system. We may also ask what leads to the flow around the entire system. It requires an extremely large value of specific angular momentum in order to have a circulatory flow around the entire system. How can this angular momentum be imparted to the matter or what is the ejection mechanism? Actually under the ordinary circumstance of ejection the motion of the envelope surrounding the entire system possesses likely a radial component. All these difficulties arise from the fact that we do not know how to impose the realistic boundary condition to the hydrodynamic equations. It follows that even though the hydrodynamic approach is a legitimate one, it has not so far provided us with any new insight to the complicated problem of gaseous flow in the binary system. 
For these reason Huang (1965a) has proposed a statistical approach based on the three-body problem. By considering the three-body problem we can take proper care of the boundary condition such as the ejection at the Lagrangian points, etc. By considering statistics of Jacobian constants of particles in the stream the collision among them are accounted for. However, so far the result obtained from this approach is no more than the particular solution that Prendergast has derived from the hydrodynamic considerations. But we still hope that something observationally significant could be obtained later by this approach.

What makes the trajectories derived from the restricted three-body problem meaningless are collisions among particles. Now it may be argued that we can find trajectories in which collisions do not play an important role. If so, such trajectories may represent some realistic case of motion of gaseous streams. What are then the conditions for such trajectories? Obviously each must be devoid of loops or cusps so that it does not intersect itself, because any intersection only means collisions. However, a single trajectory without loops and cusps is not sufficient to justify a gaseous stream that follows the trajectory. It must be stable and be surrounded by a family of similar non-intersecting trajectories such that the two adjacent ones differ only infinitesimally in all aspects. In this way we identify a gaseous stream to a family of non-intersecting trajectories. Such a condition obviously is satisfied in the case of a family of non-intersecting periodic orbits, although this is by no means the sole possibility.

Poincaré has shown that periodic orbits exist around each of the two finite bodies in the restricted three-body problem. We have developed a perturbation method for finding such orbits (Huang, 1967a). Figure 1 illustrates a few such orbits in a family around the more massive component in the orbital plane together with the innermost contact surface, corresponding to a system of the mass ratio of 4 to 1 . Indeed these orbits form a family of similar and non-intersecting trajectories in the plane of orbit. We suggest that the existence of such a family of stable periodic orbits makes gaseous rings around the component star in a binary system possible, just as the existence of periodic orbits makes rings around single stars possible. In a sense the existence of periodic orbits provides the particles with some kind of invisible tracks. Those particles that move with velocities quite different from these corresponding to the periodic orbits simply depart from the tracks. But those that move with velocities close to that corresponding to the periodic orbits are trapped in tracks and become a part of the ring system. Because small perturbations from the stable periodic orbits will remain small, particles do not move away easily once being captured in the ring. They will stay there for a long time until some drastic disturbance kicks them out of the periodic orbits and thereby destroys the ring structure. In this way we link the observed ring with the existence of a family of stable periodic orbits around the star. Perhaps most particles in Saturn's rings are moving in periodic orbits because of the long time of their existence. However the particles in the gaseous rings in Be stars are. likely moving in nearly periodic orbits, as their motions have not been left undisturbed for any long period of time.

The existence of periodic orbits circling around each component shows nothing 


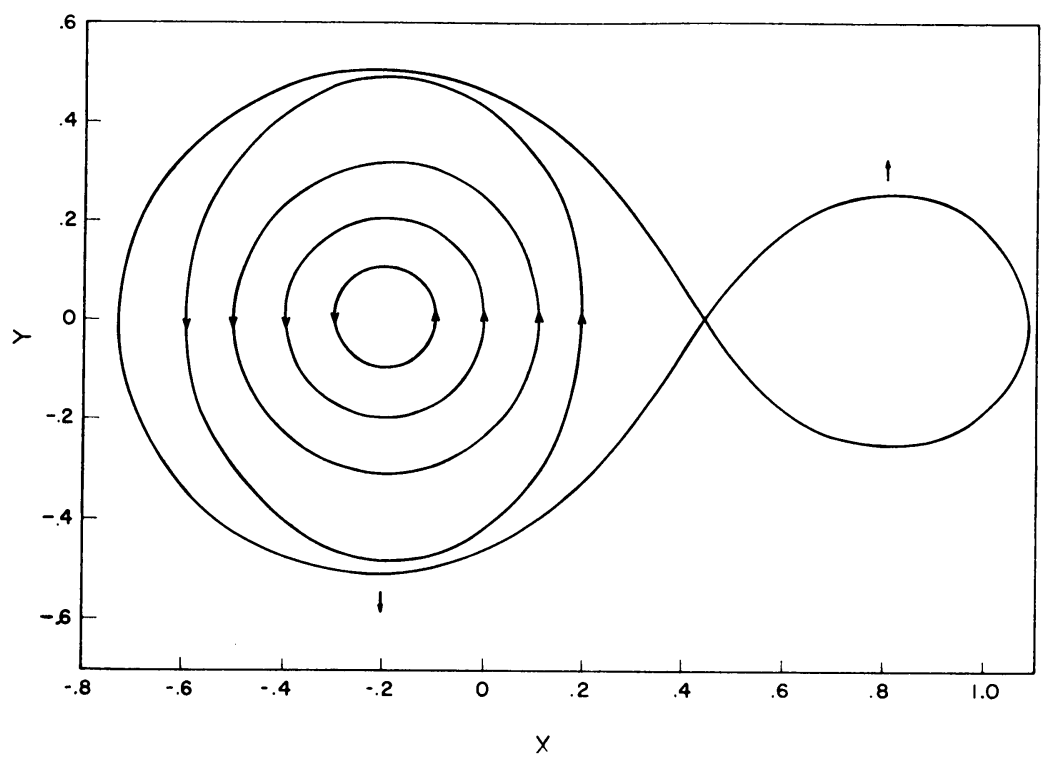

Fig. 1. The family of periodic orbits around the more massive component in a binary system. According to our interpretation the existence of such a family makes the ring formation possible. Note the increasing departure of these orbits from the circular form as their size increases.

more than the possibility of the gaseous ring. It does not explain its actual appearance. The fact that it revolves around the component star indicates the large amount of angular momentum it possesses. The question is: why does the matter possess such a large amount of angular momentum? Here we encounter a physical problem in contradistinction to the mathematical problem of periodic orbits just described.

\subsection{Physical CONSIDERations}

Several ways that rotating rings and disks may be formed in binary systems can be visualized (Huang, 1971).

(i) Rotational instability - just as the emission rings are formed around Be stars as the result of a combination of rapid rotation at the equator and radial instability, rings can be similarly formed around the components of binaries if the star is rotating rapidly. Obviously it would be the case if at least one of the components in a binary is a Be star (Hutchings and Wright, 1971). However, many emission rings found in binaries do not appear to be formed in this way, because the component stars in the center of rings are not rotating at such a rapid rate as to become rotationally unstable and most of their spectral types are too late for radial instability according to Lucy and Solomon's mechanism.

(ii) Angular momentum transport through magnetic braking - we will discuss the magnetic braking theories later. In the process the stellar angular momentum is being transferred to the surrounding medium which collapses into a rotating disk when 
enough angular momentum has been acquired. This may happen whether the star is single or a component of a binary system.

The angular momentum transport through magnetic braking takes place, most likely, in the pre-main-sequence stage of stellar evolution (see references in Huang, 1969). According to Hall (1971 a) the secondary component of BM Orionis is a case of disk formation in the pre-main-sequence stage in a binary system. While its primary B star may have already reached the main sequence, the secondary component could very well be still in the stage of gravitational contraction leading to the main sequence. Also, the cooler components in RS CVn binaries may be in pre-main-sequence contraction too (Catalano and Rodonò, 1967; Hall, 1972), although this suggestion has not been unanimously accepted (Koch, 1970). Hall (1972) further suggested that the cool star in RS CVn itself may be a T Tauri-like star. This suggestion has been obtained from a careful analysis of the light curve but does not come from spectroscopic data.

(iii) Cataclysmic ejection - when a star explodes, it leaves some debris around. If the star originally possesses a large amount of angular momentum, such a debris will easily collapse into a disk. Furthermore, if the explosion is of the supernova nature, this debris will be rich in heavy elements which may condense into solid particles. We suspect that the secondary component of $\varepsilon$ Aur may possess a disk just like this (Huang, 1965b; Kopal, 1971).

(iv) Ejection as a result of stellar evolution reaching the innermost contact surface - For the star in a binary system there is the instability caused by, on the nuclear time scale, the evolutionary expansion which brings its radius into direct contact with the Roche limit. The mass flow due to this cause can be considerable. Therefore once matter has started to flow out, the loss of mass itself may induce further instabilities not only on the nuclear time scale but also on short time scales, such as Kelvin and pulsational time scales (Morton, 1960). Considering the fact that the binary elements and the mass ratio are themselves related to the mass loss and/or gain of component stars, we are confronted with a very complicated problem, in which the time scale of dynamical evolution of the binary itself must also be taken into account. In other words, stellar evolution of component stars and dynamical evolution of the binary system are coupled together (Smak, 1962). The coupling is provided of course by the ejected matter. That makes the modes of ejection and flow exceedingly important not only for those who are interested in stellar spectroscopy by also those in stellar evolution (e.g. Paczński, 1971; Plavec, 1968).

Ejection in this catagory is expected to take place intensively, though not exclusively, at the Lagrangian points (Kuiper, 1941) and provides a cause for formation of rotating rings and disks. Because of symmetry, it is reasonable to expect that the ejection taking place at the Lagrangian points produces a medium confined to the orbital plane and its immediate neighborhood. In other words, whether around the companion of the ejecting star or around the entire system, the ejected gas will be distributed more like a disk than a sphere. The structure of the disk could be either amorphous or rotating, depending upon the amount of angular momentum the medium possesses. 
Observationally we have found cases of the rings revolving around the more massive component while the less massive component fills the Roche surface (e.g. Huang and Struve, 1956). That happens in most Algol-type binaries. It may be argued that the angular momentum per unit mass of the less massive component is greater than that of the more massive component. Thus when the mass ejected by the less massive component reaches the neighborhood of the more massive component, it finds itself possessing an extra angular momentum. Such a gaseous medium around a central field (in this case, produced by the massive component) will simply collapse into a rotating ring or disk (Huang, 1957).

The angular momentum of a particle varies as it moves in the gravitational field of two revolving stars. However, once it has entered into a periodic orbit, its average value remains constant. Consequently a definite amount of angular momentum can be assigned to each ring of gases. Thus, in order to form the ring, this amount of angular momentum must be available to the ejected matter. The latter's angular momentum is supplied by the ejecting star at the time of ejection and further supplemented by acquisition during its flight from the point of ejection to the place where the ring is formed. This is because the ejected matter being acted on by the Coriolis force, moves in such a way its angular momentum increases in general after its ejection at the inner Lagrangian point. However, if the angular momentum of the ejected matter is not sufficiently large, it may fall directly to the massive component, forming as in the case of U Cephei what Batten and Plavec (1971) term a luminous bridge. When the matter falls into the primary component, its angular momentum is transported to the atmosphere and makes the latter rotate faster than otherwise would be the case. In this way we have explained the non-synchronized rotation of the primary component of this system (Huang, 1966b). In such cases, one may visualize the gaseous ring as coalescing into the atmosphere. Such a view seems to be supported by observational results obtained by Batten (1969) who has found that the velocity from the emission lines in the spectra of $U$ Cep is virtually identical to the rotational velocity of the primary star. Thus the pictures presented by Batten, Plavec and myself are consistent.

There remains the question raised by Batten (1970) whether the two events - formation of a gaseous ring around the massive component and the driving of the atmosphere into rapid rotation by the falling gas - are coexisting or not. We agree with him in that there is no reason for the two events to be mutually exclusive. Whether the two events can actually occur side by side depends upon the mode of ejection as well as the mode of dissipation of the ring. If the ejection velocities cover a wide range, some particles will fall into the star while others form the ring. In such a case the two events occur simultaneously. During the dissipation of the ring the constituent particles may be swept into the atmosphere of the star to drive the atmospheric rotation instead of being driven away into space. In such a case the one event becomes consequential to the other. However under some different modes of ejection and dissipation, the two events could become mutally exclusive.

We have attributed formation of rotating rings mainly to the disparity of masses of two component stars. As an educated guess, rings may be formed around more massive 
components in binary systems whose mass ratio, $M_{1} / M_{2}$, is between 2 and 8 (Huang and Struve, 1956). What would happen if the mass ratio becomes greater than, say 10 ? The specific angular momentum of the matter ejected by the less massive component will be very high in such systems. But too high a value of angular momentum is not necessarily conducive to formation of the ring around the component star because the range of permissible rings is limited there. In such cases rotating rings or disks may more readily form around the entire system. Scarcity of observable systems in this range of mass ratios makes the discussion academic.

If there is not enough angular momentum present, the medium around the component star can not collapse into a regularly rotating ring or disk. Such a disk disperses more readily than the rotating one unless it is constantly replenished with new matter by ejection. Kraft $(1958,1959,1962)$ has suggested that disks exist around some eruptive components of binaries such as DQ Her, T Cor B, U Gem, etc. According to him the masses of two components in each of such systems are nearly equal. Often the disk is associated with the slightly less massive eruptive component while the slightly more massive component fills the innermost contact surface and is losing mass. Whether such disks are completely supported by the centrifugal force is at present not clear.

\subsection{ObSERVATIONAL MANifESTATIONS}

The rotating rings and disks around component stars in close binary systems will reveal their existence to us in several ways, depending upon (1) the nature of the central star, (2) the nature of the gaseous ring and disk itself. When the star is hot and luminous, and the matter in the ring is rare, emission lines of hydrogen and other elements perhaps will be the dominant feature. Indeed the gaseous emission rings around the more massive components of many eclipsing binaries have been discovered in this way. While Joy $(1942,1947)$ first proposed the existence of a gaseous ring that produces emission lines in RW Tauri, it was Struve (e.g. Sahade, 1960) who made a large number of discoveries of this kind of objects. With the use of rapid scanning techniques, such as effectively employed by Hutchings and others, the gaseous rings could be quantitatively studied. The relation between the rotational velocity of the ring and the period of the binary especially can be studied.

Let $M_{1}$ and $M_{2}$ respectively be masses of two component stars, $\left(M_{1} \geqslant M_{2}\right), a$, the semi-major axis of the relative orbit, and $\varrho$ the radius of the gaseous ring around $M_{1}$. In the two-body approximation we may assume that the ring is circular making $\varrho$ constant in all phases. However, in the approximation based on periodic orbits in the restricted three-body problem, the ring cannot be circular, as is evident in Figure 3.1. If $\varrho / a$ is to be determined by the time of eclipse of the emission line, $\varrho$ must be the radius in the direction perpendicular to the line joining two component stars: Similarly the rotational velocity of the emission ring should be constant in the twobody approximation but is variable in the three-body approximation. Therefore we specify the rotational velocity, $V$, to be at the point where $\varrho / a$ is measured. It has been shown that based on the periodic orbits in the restricted three-body problem, $V$, in 
the c.g.s. unit is given by

where

$$
V=\left(\frac{G M_{1}}{\varrho}\right)^{1 / 2} f\left(\mu, \frac{\varrho}{a}\right) \sin i
$$

$$
\mu=\frac{M_{2}}{M_{1}+M_{2}},
$$

and $G$ is the gravitational constant, and $i$ is the orbital inclination while $f(\mu, \varrho / a)$ is a function which has been given in a tabulated form (Huang, 1967a). Needless to say we have assumed that the plane of the ring coincides with the orbital plane. For small $\mu$ and small $\varrho / a$

$$
f\left(\mu, \frac{\varrho}{a}\right) \rightarrow 1,
$$

Equation (1) reduces itself to the case of the two-body approximation. Since $V$ and $\varrho / a$ are measurable, we have from Equation (1) a relation between total mass, the mass ratio and the separation. If $K_{1}$ denotes the semi-amplitude of the velocity curve of $M_{1}$, and $e$ the eccentricity of the binary orbit, the relation between $V$ and $K_{1}$ is given by

$$
\left(\frac{V}{K_{1}}\right)^{2}=\frac{M_{1}\left(M_{1}+M_{2}\right)}{M_{2}^{2}}\left(1-e^{2}\right)\left(\begin{array}{l}
a \\
\varrho
\end{array}\right) f^{2}\left(\mu, \frac{\varrho}{a}\right),
$$

which determines the mass ratio of the system even when we do not have the radial velocity curve of $M_{2}$

Thus the motion of the gaseous particles in the ring gives us some information about the mass ratio as good as the motion of $M_{2}$ itself. However since $f(\mu, \varrho / a)$ has been determined on the assumption of the restricted three-body problem we can apply this method for determining the mass ratio only for systems with small eccentricities. For binaries of large eccentricities one can make similar calculations as we did for circular orbits. In such cases the function $f(\mu, \varrho / a)$ should be replaced by another one containing $e$ as an additional variable. In reality there cannot be many binaries of high. eccentricities which possess rings because of their large disturbing effect on the stability of the ring.

Struve (1950) has found that the rotational velocity of the gaseous ring is related to the orbital period, $\dot{P}$, of the binary in the following way

$$
V^{3} \propto \frac{1}{P}
$$

Within the restricted three-body approximation such a relation would mean that

$$
\varrho_{a}^{\varrho} \propto M_{1}^{2 / 3}\left(\frac{M_{1}}{M_{1}+M_{2}}\right)^{1 / 3} f^{2}\left(\mu, \frac{\varrho}{a}\right)
$$

Batten (1972) has pointed out that most of the observational data available to 
Struve concerned Algol-type systems, in which indeed $M_{1}$ and $M_{1} /\left(M_{1}+M_{2}\right)$ do not vary greatly. With data now available for other kinds of binaries he has found that they all fit a somewhat different relation namely

$$
V^{4.6} \propto \frac{1}{P}
$$

On the basis of the restricted three-body approximation, it can be easily shown that this relation indicates that

$$
\stackrel{\varrho}{a} \propto \frac{1}{a^{0.35}}\left(\frac{M_{1}}{M_{1}+M_{2}}\right)^{0.22} M_{1}^{0.78} f^{2}(\mu, \stackrel{\varrho}{a}) .
$$

Hence $\varrho / a$ seems to be related not only to the mass and the mass ratio as in relation (6) but also on the size of the binary. Since small masses often occur in small systems, the effect of $M_{1}$ and $a$ may cancel out and leave $\varrho / a$ not greatly different from very small to very large systems. Thus, the relation given by relation (7) and the narrow range of $\varrho / a$ in systems of greatly different sizes both pointed out by Batten in this symposium, represent the two facts that are closely related. Both show that the mass of the star around which the gaseous ring revolves increases with the separation of the two components in the binary.

Finally it must be said that the present analysis is tentative at best. In the first place the ring cannot be geometrically thin as assumed in the calculation. Secondly, there is also the question of whether the matter in the disk is completely supported by the centrifugal force. However, the suggestion that the statistical relation obtained by Batten indicated a mass-separation relation among those binaries included in his statistical study has the intuitive appeal.

It is reasonable to expect that almost all kinds of variation in emission found in $\mathrm{Be}$ stars can happen in binaries with rings. However, in most binaries the emission is too weak to be observed outside eclipse. Consequently such variations have not been recognized because they are overshadowed by the intensity variation caused by eclipse itself.

If the matter in the disk increases, we would expect that the continuous opacity, say due to electron scattering, will be considerable and the disk may become semitransparent. Thus instead of emission, the disk will reveal itself by its obscuration. It was this argument that led us to suggest that the secondary component of $\beta$ Lyrae system may possess just such a structure (Huang, 1963a). Indeed, it seems that all essential features of this peculiar system can be understood in terms of this structure. More recently, Hall (1971a) has shown that the secondary of BM Orionis may also possess a semi-transparent disk of this kind.

We may further argue that the rotating gaseous disk exists in such a state that it both produces emission lines and causes obscuration of the companion star. This situation can arise if the density of gas is rare at some places (likely near the edges) which produces emission, but dense at other places (likely in the middle) where obscurtion occaurs. Hall (1971b) suggested that RS Cephei may represent an example for 
such an intermediate case. Hall and Garrison(1972) have reported SW Cygni as another example.

If the star is not too hot or the gaseous ring is far away from the star, the matter in the disk may condense into solid particles or planetesimals. In such a case, the presence of the disk could be detected by its emission in infrared radiation as well as its obscuration of the secondary component. The secondary component of $\varepsilon$ Aur may possess such an opaque disk. If so, the disk would be in many respects like the solar nebula (Huang, 1965b; Kopal, 1971) and may even develop into a planetary system.

However the interpretations of the light curve of $\varepsilon$ Aur based on a semi-transparent disk that is composed of solid particles has its difficulty. We have mentioned infrared emission as one of the characteristics of such a disk. However, Low and Mitchell (1965) found no infrared excess in this system. Therefore at present we can only take the suggested disk composed of solid particles in $\varepsilon$ Aur as tentative. As we have emphasized elsewhere (Huang, 1971), a critical test of this hypothesis will be detection of eclipse in infrared when the primary component, which may be deficient in infrared radiation and thereby obliterate the infrared emission of the disk, obscures the latter from the observer. We have found from the disk model that the next infrared eclipses would occur in June 1974-June 1976 according to the spectroscopic orbital elements given by Kuiper et al. (1937) or in October 1971-October 1973 according to those given by Morris (1962). Since this is a very critical test, we hope that some infrared astronomers will observe this star in the next few years.

Apart from the circulatory motions that are associated with the ring and disk structure and the jet motion starting from the Lagrangian points, there must be other kinds of flow taking place in the binary system. We have already mentioned those possibilities in Section 2.2. At distances large compared with the separation between the two components, perhaps the flow pattern may not be greatly different from that around the rotating star. The matter which must have a high concentration in the orbital plane and decrease rapidly as we move away from it is likely in a state of expansion as has been observed in $\beta$ Lyrae (e.g. Struve, 1958). The tangential velocity would depend upon how effective the angular-momentum transfer is from the binary system to the medium.

An intriguing question that is related to the gaseous flow in the binary system is the change in the orbital period. As soon as there is gaseous flow in the system the orbital period will change. Hence the change in orbital period in close binaries is the general rule instead of the exception because some kind of flow is inevitable in close binaries. In general such a change is small and may not be observationally detected. In those cases where the variation in period has been detected, it is also difficult to interpret them physically because the variation depends on the total mass involved in the flow, the mode of ejection and flow pattern and many other factors not connected with mass flow (e.g., Kruszewski, 1966). Huang (1963b) has expressed the change of period in terms of specific energy and specific angular momentum - a practice that has been followed by Piotrowski (1964). By doing so the period changes in a few simple, but nevertheless realistic, cases have been treated. On the other hand Van 't Veer's (1972) 
recent study of period change of contact binaries has imposed some different assumptions on the modes of mass ejection and flow. In any case the mode of ejection and flow must be assumed before the period change can be calculated. As we have not solved the flow problem in a binary system it is premature to undertake a general treatment of period change, although a serious attempt in this direction has been made by Piotrowski (1964; also Kruszewski, 1966). Therefore we may conclude that since there is no way at present to ascertain the exact mode of ejection and flow pattern that actually takes place in any particular system, the rate of mass change and/or loss derived from the rate of change in period is tentative.

\section{Consequences of Ejection by Stars with Magnetic Fields}

The mass of gaseous streams around stars is usually very small compared with the star itself. But observationally they play a critical role since the streams, being outside the photosphere, are exactly what will imprint their marks on the light we observe. Thus, gas streams with their low densities are the cause of many peculiarities found both spectroscopically and photometrically, and a complete understanding of stellar spectra, especially those of peculiar ones, must include the behavior of the gaseous stream around the stars, whether they are single or in binary systems.

In spite of the clear marks that are imprinted on stellar spectra, the ejected matter plays in general an insignificant role in shaping the over-all structure of the ejecting star itself. However, when the star also possesses a magnetic field, the ejection process could modify the nature of the star considerably. We shall discuss the following three cases that belong to this category.

\subsection{BRAKING OF STELLAR ROTATION}

One of the most puzzling phenomena in stellar astronomy is the discontinuity of the rotational behavior of the single main-sequences stars, discovered by Struve (1930). While rotation is common among early-type stars, stars later than spectral division F5 have never been found to rotate in any measurable way. That puts the equatorial velocities of the late type stars less than $5-10 \mathrm{~km} \mathrm{~s}^{-1}$ at the present accuracy of determination.

It is difficult to understand this peculiar behavior of stellar rotation when one considers the fact that whatever is the mechanism of star formation, it is always accompanied by acquisition of some angular momentum. It is hard to find a plausible reason for such a discontinuity in stellar angular momenta at the time of star formation. Several theories (See Kraft, 1970, for a general review) have been proposed in order to explain this strange phenomenon. In general it has been thought that this behavior is likely a result of braking of stellar rotation after the star has been formed. If so, there are three questions that we must answer. First, what is the braking mechanism? Second, what is the observational evidence for such a mechanism? And finally, why does it brake only the late-type stars? When we reviewed stellar rotation (Huang and Struve, 1960), none of the three questions could be satisfactorily answered. 
But in the past decade new developments in different fields all lead us to believe that magnetic activities occur in the early stage of stellar evolution. General reviews of such developments have been given elsewhere (Huang, 1969; 1973). Therefore it is reasonable now to say that the braking of stellar rotation is of electro-magnetic nature. However, there are many ways that braking of stellar rotation can be effected by the magnetic field. We have mentioned already one by Lüst and Schlüter (1955) in connection with Hazlehurst's theory of ring formation. It would be out of the scope of this paper to present theories of magnetic braking so far advanced, all of which concern the motion of gases in the stellar neighborhood. Suffice it to say here that all theories are in principle attractive as far as the braking is concerned. But most require some assumptions which will lead to results contrary to what has actually been observed. It is our judgement that Schatzman's (1962) theory of flare-like activities seems to have the benefit of observational support. According to him when a jet of ionized gas is ejected during flares from the stellar surface, the matter is forced to turn with the stellar magnetic field up to a distance until the matter breaks away. He has applied this idea to the early stage of stellar evolution when the star is dominantly convective and therefore it is reasonable to expect magnetic activities on the surface just as the solar magnetic activity is attributed to the convection and/or differential rotation of the Sun.

This theory is consistent with the evidence derived from observation. In the first place it has been known that $\mathrm{T}$ Tauri and T Tauri-like stars which are in the premain-sequence stage are indeed ejecting matter (e.g. Herbig, 1962; Kuhi, 1964, 1966). Flares are often observed in some of these st ars. According to Herbig (1952), T Tauri stars are indeed rotating with appreciable velocities, indicating that the magnetic braking process is not yet completed.

Schatzman's theory was proposed without the benefit of Hayashi's (1961) theory of pre-main-sequence evolution that has now been generally accepted. In the light of Hayashi's theory, Schatzman's argument for the differentiation of rotational behavior at F5 can no longer be applied. However his basic assumption that convection is the source of flares is still attractive. Therefore we may slightly modify his theory to assume that the braking of rotation takes place in the Hayashi phase of stellar evolution (Huang, 1967b). In massive stars the Hayashi phase is short. Consequently the rotation has not been greatly braked. While in less massive stars the Hayashi phase is long. Consequently the rotation has been effectively braked. We have proposed this explanation more on intuitive grounds than on actual calculation. But results of recent calculations by Larson $(1969,1972)$ have fully confirmed our intuitive suggestion because he has found that stars of masses greater than $1.5 \odot$ never undergo the Hayashi phase. Instead they first appear on the radiative pre-main-sequence track. His results provide a strong support to our hypothesis that braking of rotation occurs in the Hayashi phase, because the limiting mass of $1.5 \odot$ he has derived is very close to the $1.3 \odot$ that corresponds to the mass of stars of spectral type F5, where the sudden change of rotational behavior occurs. The small difference could be the result of uncertainty either in the calibration of stellar masses or in the computation. 


\subsection{FORMATION OF PLANETARY SYSTEMS IN THE UNIVERSE}

Schatzman's (1962) paper is devoted solely to the braking of stellar rotation through mass ejection during flare-like activities. He assumes that the ejected matter escapes from the star, carrying away the angular momentum to infinity. Actually one may argue (Huang, 1967b; Nakano, 1970) that when the star is formed from the interstellar medium, there must be left a remnant or residue matter in its neighborbood. If so, those ejected particles will mix up with or stir the residue medium and impart their angular momentum to it. Once the medium has acquired the angular momentum, it naturally collapses into a rotating disk in which the gases will condense to form grains and grains will accret to become planetesimals and finally planets themselves. In this way we arrive quite naturally at a system that has all the major features of our own planetary system, such as the near-circular and near co-planar nature and the disparity in the angular momentum distribution between the Sun and the planets. Since, according to our reasoning, the disk is formed as a result of braking, it follows that planetary systems probably accompany most main-sequence stars of spectral type later than F5. According to this theory planetary systems are being formed at the pre-main-sequence stage.

\subsection{Formation OF CLOSE BINARIES OF THE W URSAE MAJORIS TYPE}

If the rotational angular momentum can be reduced by magnetic braking, the orbital angular momentum of a binary system can be similarly dissipated. Consequently as long as the braking mechanism continues, the separation between two components of the system will gradually decrease until the two become physically in contact (Huang, 1966a; Mestel, 1967). Such a process may be the cause for forming W U Ma stars. This theory has the attraction in that $\mathrm{W} \mathrm{U} \mathrm{Ma} \mathrm{systems} \mathrm{are} \mathrm{mainly} \mathrm{composed}$ of stars of spectral type $F$ and later, just in the spectral range where rotation is effectively braked. Hence we are able to invoke the same mechanism for explaining the very slow rotation of stars later than F5 and the high abundance of W U Ma systems.

What would happen if the contacting binary is further braked? It can be easily seen that mass will flow from the less to the more massive component. As an extreme case the less massive component could be completely absorbed, by what may be called a fusion process, in the more massive component. In such a case the less massive component turns inside out. The matter in its core becomes the atmosphere of the merged star. If thermonuclear reactions of converting hydrogen into helium have already started in the interior of the less massive component, such a mode of mass transfer will result in an overabundance of helium in the atmosphere of the final star (Huang, 1966a).

This theory of the origin of contact binaries has its weakness in that while contact binaries are indeed composed mainly of stars of spectral type $F$ and later, there are contact binaries with components of earlier spectral types. For this latter group of binaries, it is difficult to apply the magnetic braking idea. Consequently we may have 
to conclude that magnetic braking is not the sole agency for making contact binaries, although it may play a dominant role in shaping up W U Ma systems.

\section{Acknowledgments}

I sincerely thank Dr. Batten for sending me beforehand the draft of his paper to be presented in this syposium. As has been mentioned in the text, Dr. Batten's paper induced me to discuss the velocity-period relation of rings in binaries which would not otherwise be discussed here. Also I take pleasure in thanking Dr. Hall who sent me the reprint of a paper on S W Cygni and Dr. Van 't Veer who sent me the reprint of a paper on period changes in contact systems, before their publication. Preparation of this article has been supported by a grant from the National Aeronautics and Space Administration.

\section{References}

Batten, A. H.: 1969, Publ. Astron. Soc. Pacific 81, 904.

Batten, A. H.: 1970, Publ. Astron. Soc. Pacific 82, 574.

Batten, A. H.: 1972, presented at this symposium (p. 1).

Batten, A. H. and Plavec, M.: 1971, Sky Telesc. 42, 147 and 213.

Buerger, P.: 1969, Astrophys. J. 158, 1151.

Carruthers, G. R.: 1968, Astrophys. J. 151, 269.

Catalano, S. and Rodono, M.: 1967, Mem. Soc. Astron. Ital. 38, 395.

Crampin, J. and Hoyle, F.: 1960, Monthly Notices Roy. Astron. Soc. 120, 33.

Crampton, D. and Hutchings, J. B.: 1972, Nature 237, 92.

Curtiss, R. H.: 1926, J. Roy. Astron. Soc. Can. $20,19$.

Hall, D. S.: 1971a, Veröffentl. Remeis-Sternw. Bamberg 9, 217.

Hall, D. S.: 1971b, IAU Colloquium No. 16 (in press).

Hall, D. S.: 1972, Publ. Astron. Soc. Pacific 84, 323.

Hall, D. S. and Garrison, L. M., Jr.: 1972, Publ. Astron. Soc. Pacific 84, 552.

Hardorp, J. and Strittmatter, P. A.: 1968, Astrophys. J. 153465.

Hayashi, C.: 1961, Publ. Astron. Soc. Japan 13, 450.

Hazlehurst, J.: 1967, Z. Astrophys. 65, 311.

Herbig, G. H.: 1952, J. Roy. Astron. Soc. Can. 46, 222.

Herbig, G. H.: 1962, Adv. Astron. Astrophys. 1, 47.

Huang, S.-S.: 1957, J. Roy. Astron. Soc. Can. 51, 19.

Huang, S.-S.: 1963a, Astrophys. J. 138, 342.

Huang, S.-S.: 1963b, Astrophys. J. 138, 471.

Huang, S.-S.: 1965a, Astrophys. J. 141, 201.

Huang, S.-S.: 1965b, Astrophys. J. 141, 976.

Huang, S.-S.: 1966a, Ann. Astrophys. 29, 331.

Huang, S.-S.: 1966b, Ann. Rev. Astron. Astrophys. 4, 35.

Huang, S.-S.: 1967a, Astrophys. J. 148, 793.

Huang, S.-S.: 1967b, Astrophys. J. 150, 229.

Huang, S.-S.: 1969, Vistas Astron. 11, 217.

Huang, S.-S.: 1971, IAU Colloquium No. 16 (in press).

Huang, S.-S.: 1972, Astrophys. J. 171, 549.

Huang, S.-S.: 1973, to be published.

Huang, S.-S. and Struve, O.: 1956, Astron. J. 61, 300.

Huang, S.-S. and Struve, O.: 1960, in J. L. Greenstein (ed.), Stellar Atmospheres, Chicago Univ. Press, Ch. 8.

Hutchings, J. B.: 1970, Monthly Notices Roy. Astron. Soc. 150, 55.

Hutchings, J. B. and Wright, K. O.: 1971, Monthly Notices Roy. Astron. Soc. 155, 203. 
Jeans, J. H.: 1925, The Dynamical Theory of Gases, Cambridge Univ. Press, Cambridge, Ch. 15. Johnnson, M.: 1958, in 'Etoiles à raies d'émission', Mem. Soc. Roy. Sci. Liège, 4ème Ser. 20, p. 219. Joy, A. H.: 1942, Publ. Astron. Soc. Pacific 54, 35.

Joy, A. H.: 1947, Publ. Astron. Soc. Pacific 59, 171.

Koch, R. H.: 1970, in K. Gyldenkerne and R. M. West (eds.), 'Mass Loss and Evolution in Close Binaries', Proc. IAU Colloquium No. 6, Copenhagen Univ. Copenhagen, p. 65.

Kopal, Z.: 1971, Astrophys. Space Sci. 10, 332.

Kraft, R. P.: 1958, Astrophys. J. 127, 625.

Kraft, R. P.: 1959, Astrophys. J. 130, 110.

Kraft, R. P.: 1962, Astrophys. J. 135, 408.

Kraft, R. P.: 1970, in G. H. Herbig (ed.), Spectroscopic Astrophysics, Univ. of California Press, Berkeley, p. 385.

Kruszewski, A.: 1966, Adv. Astron. Astrophys. 4, 233.

Kuhi, L. V.: 1964, Astrophys. J. 140, 1409.

Kuhi, L. V.: 1966, Astrophys. J. 143, 991.

Kuiper, G. P.: 1941, Astrophys. J. 93, 133.

Kuiper, G. P., Struve, O., and Strömgren, B.: 1937, Astrophys. J. 86, 570.

Larson, R. B.: 1969, Monthly Notices Roy. Astron. Soc. 145, 271.

Larson, R. B.: 1972, Monthly Notices Roy. Astron. Soc. 157, 121.

Limber, D. N.: 1967, Astrophys. J. 148, 141.

Limber, D. N.: 1969, Astrophys. J. 157, 785.

Limber, D. N.: 1970, in A. Slettebak (ed.), Stellar Rotation, Gordon and Breach, New York, p. 274.

Limber, D. N. and Marlborough, J. M.: 1968, Astrophys. J. 152, 181.

Low, F. J. and Mitchell, R. I.: 1965, Astrophys. J. 141, 327.

Lucy, L. B. and Solomon, P. M.: 1970, Astrophys. J. 159, 879.

Lüst, R. and Schlüter, A.: 1955, Z. Astrophys. 38, 190.

McLaughlin, D. B.: 1943, Publ. Michigan Obs. 8, 149.

McLaughlin, D. B.: 1950, Pop. Astron. 58, 50.

McLaughlin, D. B.: 1961, J. Roy. Astron. Soc. Can. 55, 13 and 73.

Merrill, P. W. and Burwell, C. G.: 1933, Astrophys. J. 78, 87.

Mestel, L.: 1959, Monthly Notices Roy. Astron. Soc. 119, 249.

Mestel, L.: 1967, in 'Instabilité Gravitationelle et Formation des Étoiles, des Galaxies, et leurs Structures Caractéristiques', Mem Soc. Roy. Sci. Liège, Sème Ser. 15, p. 351.

Morris, S. C.: 1962, J. Roy. Astron. Soc. Can. 56, 210.

Morton, D. C.: 1960, Astrophys. J. 132.146.

Nakano, T.: 1970, Prog. Theor. Phys. 44, 77.

Öpik, E. J.: 1963, Geophys. J. Roy. Astron. Soc. 7, 490.

Paczński, B.: 1971, Ann. Rev. Astron. Astrophys. 9, 183.

Parker, E. N.: 1963, Interplanetary Dynamical Processes, Interscience Publ., New York.

Piotrowski, S. L.: 1964, Acta Astron. 14, 251.

Plavec, M.: 1968, Adv. Astron. Astrophys. 6, 201.

Pottasch, S.: 1959, Ann. Astrophys. 22, 297.

Prendergast, K. H.: 1960, Astrophys. J. 132, 162.

Sahade, J.: 1960 in J. L. Greenstein (ed.), Stellar Atmospheres, Univ. of Chicago Press, Chicago, Ch. 12.

Schatzman, E.: 1962, Ann. Astrophys. 25, 18.

Slettebak, A.: 1966, Astrophys. J. 145, 126.

Smak, J.: 1962, Acta Astron. 12, 28.

Spitzer, L., Jr.: 1952, in G. P. Kuiper (ed.), The Atmospheres of the Earth and Planets, Univ. of Chicago Press, Chicago, Ch. 7.

Struve, O.: 1930, Astrophys. J. 72, 1.

Struve, O.: 1931, Astrophys. J. 73, 94.

Struve, O.: 1941, Astrophys. J. 93, 104.

Struve, O.: 1950, Stellar Evolution, Princeton University Press, Princeton.

Struve, O.: 1958, Publ. Astron. Soc. Pacific 70, 5.

Togure, T.: 1969, Astron. Astrophys 1, 253.

Van 't Veer, F.: 1972, preprint. 